Original article

\title{
The effect of emotional intelligence training on employed nurses
}

\author{
Ali Vahidi Sabzevar ${ }^{1, *}$, Hamid Robat Sarpoosh², Farzaneh Esmaeili ${ }^{3}$ Amir Khojeh ${ }^{4}$
}

(Received: 19 Mar 2016; Accepted: 3 Jul 2016)

\begin{abstract}
Background and Purpose: Nurses are frequently exposed to anxiety-related problems. In fact, anxiety and stress can negatively affect nurses' mental health and performance. Therefore, in the present study, we aimed to investigate the effect of emotional intelligence training on employed nurses in Sabzevar, Iran in 2014.

Methods: In this experimental trial, 135 nurses working in Sabzevar hospitals were enrolled and randomly assigned to intervention and control groups. The subjects were evaluated, using a demographic questionnaire, Bar-On's emotional quotient inventory, and Spielberger's State-Trait Anxiety Inventory (STAI). The intervention group was trained in six sessions by an expert. In the final session, the questionnaires were completed by the participants and analyzed. For statistical analysis, t-test and non-parametrical tests were performed. P-value less than 0.05 were considered statistically significant.

Results:Based on the findings, $80 \%$ of the samples were female. The mean age of the participants was $32.11 \pm 6.68$ years. Before the intervention, the difference between the two groups was insignificant $(P>0.05)$, while after the intervention, a negative relationship was found between the STAI score and emotional intelligence in the two groups. Also, the difference between the groups regarding STAI scores was significant $(P<0.003)$. Following the intervention, the emotional intelligence scores were higher in comparison with the preintervention period in the intervention group. Also, after the intervention, anxiety decreased in the intervention group in comparison with the pre-intervention period $(P<0.001)$.

Conclusion: The present results showed that emotional intelligence scores could improve as a result of training, while the STAI scores significantly decreased.
\end{abstract}

Keywords: Emotional intelligence, Nurses, State anxiety, Trait anxiety, Training

\section{Introduction}

Critical care nurses work in a unique environment where they are expected to make critical decisions, while facing highly stressful conditions and ethical dilemmas on a daily basis. The physical and emotional burden on nurses is rising due to changes in the demands of clients and organizations, limited availability of resources, and the increasing number of acutely ill patients; these factors may in fact lead to the burnout of critical care nurses. On the other hand, burnout can lead to emotional distress, emotional labor, depersonalization, feelings of failure, stress-related diseases, demotivation, job dissatisfaction, decreased quality of care, and conflicts with other staff members and patients (1).

According to numerous studies, work-related tension, along with mental and physical stress, can result in health disorders, functional disabilities, communication problems, low quality of care, job

\footnotetext{
1,* Corresponding author: North East Petroleum Industry Health Organization, Sarakhs, Iran. Email: Vahidiali86@yahoo.com

${ }^{2}$ Faculty of Nursing and Midwifery, Sabzevar University of Medical Sciences, Sabzevar, Iran

${ }^{3}$ Islamic Azad University, Sabzevar, Iran

${ }^{4}$ North East Petroleum Industry Health Organization, Sarakhs, Iran
} 
dissatisfaction, and job abandonment (2). Based on previous studies, $7.4 \%$ of nurses are absent from work during the week as a result of job burnout or anxiety; this rate has been reported to be $80 \%$ higher than other occupations (3).

Considering the unavoidability of some stressful factors in nursing and the need to prevent the adverse mental and behavioral effects of stress, health organization authorities should take some measures to improve nurses' work conditions and provide training on different analytical methods (4). Overall, nursing can be a stressful profession, as nurses need to deal with crucial matters such as the patients' life, death, and health (5).

There are different methods to reduce stress among nurses, including emotional intelligence training (6). Emotional intelligence, which is defined as a set of abilities for perceiving, using, understanding, and managing emotions, has been associated with a higher psychosocial adjustment (7). In other words, the way one confronts important or unimportant events in life determines his/her emotional intelligence.

Individuals with low emotional intelligence are expected to have less adjustability with stressful events and are consequently more prone to depression. In contrast, individuals with high emotional intelligence show more adjusted reactions to unfavorable life events. In addition, these individuals make decisions which less expose them to unfavorable events, and therefore, they experience lower levels of stress (8).

According to previous studies, individuals with emotional maladjustment have difficulty discerning their emotions and react to emotional situations in a negative manner. As a result, they face problems in managing and controlling these situations and experience excessive emotions (9-11). Through adjusting emotional intelligence, one can have lower levels of stress and adjust to mental tension (12-17).

Numerous stressors in the workplace can exert excessive stress on nurses. Also, these stressors can negatively affect the nurses' mental health and performance. It is possible to prevent these negative effects through emotional intelligence. Individuals with higher emotional intelligence express their feelings and emotions more freely than others.
Therefore, they receive more social support from a more extensive social network; this can in turn lead to improved mental health and invulnerability to stress.

Previous investigations have shown that emotional intelligence can affect stress $(8,18)$. In fact, emotional intelligence has a direct relationship with positive emotions (e.g., enjoyment, love, and enthusiasm) and a reverse relation with negative emotions (e.g., anxiety, hostility, and dissatisfaction) (8). In the available training programs, recognition of emotions, their interaction with an individual's internal and external dimensions, acceptance of emotions, and more importantly, correct organization of these emotions have been emphasized. Therefore, high emotional intelligence can have a positive impact on stress control and relief. Moreover, it can help improve nurses' mental health and prevent the negative personal and social effects of anxiety and stress (19).

Stress among nurses can result in a qualitative and quantitative decline in the provision of healthcare services; In addition, it has unfavorable effects on the nurses' social health. Considering the involvement of nurses in healthcare teams, their continuous interaction with the sick, their sensitive caring role, and the importance of stress prevention and control in improving the quality of care, recognition of stressful factors can be an effective step towards designing suitable plans to decrease stress among the nursing staff and improve the quality of nursing services (20-23).

After identifying the stressful factors and their significance, it is crucial to find ways to reduce or confront these stressors and eliminate their adverse effects on nurses' performance. As the positive effects of emotional intelligence have been confirmed, training can be used to decrease stress in the nursing staff and improve their performance.

With this background in mind, in this study, we aimed to determine the effect of emotional intelligence training on reducing stress among employed nurses in Sabzevar hospitals. In this research, we highlight the need for effective methods to help reduce stress among this caring staff and improve their performance through determining 
their emotional intelligence and stress level.

\section{Materials and Methods}

\section{Data collection tools}

The State-Trait Anxiety Inventory (STAI) is a commonly used tool for the evaluation of trait and state anxiety (24). This instrument can be used in clinical settings to identify anxiety and distinguish it from depressive syndromes. It is also often used in research as an indicator of distress among caregivers (25).

Form Y, which is the most popular version of STAI, consists of 20 items on trait anxiety and 20 items on state anxiety. State anxiety items include: "I am tense; I am worried" and "I feel calm; I feel secure." Also, trait anxiety items include: "I worry too much over something that really doesn't matter" and "I am content; I am a steady person." All the items are rated on a four-point scale (ranging from "almost never" to "almost always"). In this scale, higher scores indicate greater anxiety.

STAI is suitable for those who have at least a sixth-grade reading level (26 -28). In a previous study, the internal consistency coefficients for this scale ranged from 0.86 to 0.95 . Also, test-retest reliability coefficients were reported to range from 0.65 to 0.75 over a two-month interval $(29,30)$. In the present study, test-retest coefficients for this scale ranged from 0.69 to 0.89 . Overall, substantial evidence attests to the construct and concurrent validity of this scale.

After finding the norms based on previous studies, the Bar-On's emotional quotient inventory was used, which consists of 90 questions on 15 components of emotional intelligence. The norms of this questionnaire have been reported in Iran. In a previous study, the reliability of this scale was reported to be $69-86 \%$, using Cronbach's alpha (mean $=76 \%$ ) (28). Also, according to a previous study, Cronbach's alpha of this scale was $89 \%$, while its test-retest reliability was estimated at 77\% over a four-week interval $(20,25)$.

In this study, Cronbach's alpha was calculated to determine the reliability of the questionnaires. The reliability of Spielberger's STAI was estimated at $77-81 \%$, which shows that the questions were quite homogeneous. Also, the reliability of BarOn's questionnaire was $78 \%$, thus confirming its reliability. The items were rated on a five-point Likert scale (complete agreement: 5, complete disagreement: 1). The minimum score on the test was 90 , while the maximum score was 450 ; for each component, the scores ranged from 3 to 60 .

The research units were asked to fill out the questionnaires with complete relaxation and sincerity. The trainees were asked to keep their answers confidential; at the end of the research, the test results were announced. The study population in this experimental study, which was performed in hospitals affiliated to Sabzevar University of Medical Sciences in 2014, included all the employed nurses. A total of 135 samples who met the inclusion criteria (e.g., not having any mental problems or diseases, receiving no training in this area, not being pregnant, and not taking any tranquilizers) were selected.

At the beginning of sampling, the subjects were divided into control $(n=73)$ and intervention $(n=62)$ groups through simple random sampling. After taking written informed consents from the samples, a questionnaire including age, gender, educational level, work experience, work shift, and settlement status was completed. Afterwards, the 20-item STAI and the 90-item Bar-On's emotional quotient inventory were completed by the participants.

The study protocol was approved by the Ethics Committee of Sabzevar University of Medical Sciences (Medsab.Rec.93.5), and permission was obtained from the hospital officials. Moreover, objectives of the study were explained to the participants, and written informed consents were obtained from all the patients prior to the study. The participants were allowed to withdraw from the study at any time, and the researchers made sure that the study objectives were in line with the values and beliefs of the participants. Also, the researchers were obliged to inform the officials and the participants about the results of the study.

First, all the questionnaires were completed by all the participants. The subjects' emotional intelligence and STAI scores were calculated prior to grouping (and the intervention), using methods previously 
discussed. Then, through simple random sampling, after selecting the intervention group, the subjects were informed about the group to which they were allocated via an invitation letter; also, they were notified about the time and place of training. The participants were aware of the fact that they would be eliminated from the study if they failed to attend half of the sessions (three sessions).

The intervention group received emotional intelligence training in six two-hour sessions in form of conference and Q\&A sessions with psychological experts and the researcher, using speech and pamphlets. At the end of training, the two groups were asked to complete the questionnaires of emotional intelligence and anxiety.

After making the necessary coordination with the educational supervisor, continuous training was performed at Sabzevar University of Medical Sciences in two-hour sessions (re-education code: 192159303). The training sessions were held in the conference hall of Vasee Hospital over six continuous weeks. These two-hour sessions were held every week (8-10 a.m.) and were as follows:

First session: The participants were introduced to each other and were notified about the method and structure of the sessions. The subjects' expectations from the training program were identified, and the questionnaires were completed by the intervention group.

Second session: Emotional intelligence and its components (15 components of emotional intelligence scale, especially stress relief plans) were trained and discussed. First, the cases were trained on methods of adjustment with stressful factors and surroundings.

Third session: The subjects were acquainted with the concept of emotional self-discipline, expression of feelings and affection, and methods of changing instilled concepts.

Fourth session: Emotional self-control, priority of impartiality over personal beliefs, coping abilities, and relaxation therapy were taught to the participants.

Fifth session: Emotional self-provocation was highlighted during this session.

Sixth session: The subjects were trained on emotional refinement, different emotional intelligence techniques, and use of emotional intelligence; then, they were asked to fill out the questionnaires. By the end of every session, the participants' names were written down. The sessions ended after responding to the subjects' questions. The educational slides, papers, and charts were presented to the participants for practice and learning. At the end of the intervention, both the intervention and control groups completed the questionnaires. The questionnaires were collected and information, including emotional intelligence and STAI scores, was extracted, as described above.

For statistical analysis, independent t-test, paired t-test, and non-parametric tests including Chisquare and analysis of variance were performed, using SPSS version 20. In this research, P-value less than 0.05 were considered statistically significant.

\section{Results}

In total, 135 nurses participated in this study, $83 \%(n=112)$ of whom were female. Also, 104 $(77 \%)$ out of 135 nurses were married. Based on the findings, 125 (92.6\%) nurses had a bachelor's degree in nursing, and the majority $(\mathrm{n}=129,95.6 \%)$ were from Sabzevar (Table 1).

The results showed that the average age of the study population was 32.11 years. At the beginning of the study, the emotional intelligence scores in the intervention and control groups were 319.0634.02 \pm and 333.7631.62 \pm , respectively. On the other hand, following the intervention, the scores were $350.1129 .67 \pm$ and $330.4943 .84 \pm$ in the intervention and control groups, respectively.

Before the intervention, the total anxiety scores in the intervention and control groups were 84.9515.76 \pm and 77.6113.77 \pm , respectively, while after the intervention, the corresponding scores were 72.9813.55 \pm and 79.5215.68 \pm , respectively; the difference was found to be statistically significant $(P<0.001)$ (Table 2$)$. The results showed a significant difference in total anxiety before and after the intervention in the two groups $(P>0.001)$. Also, comparison of state and trait anxiety and emotional intelligence before and after the 
Table 1. The absolute and relative frequency of qualitative variables in the study population

\begin{tabular}{|c|c|c|c|}
\hline Variables & & Frequency (\%) & Total \\
\hline \multirow{2}{*}{ Gender } & Female & $112(83)$ & \\
\hline & Male & $23(17)$ & 135 \\
\hline \multirow{2}{*}{ Educational level } & BSN student & $125(92.6)$ & \multirow{2}{*}{135} \\
\hline & MSc student & $10(7.4)$ & \\
\hline \multirow{2}{*}{ Marital status } & Single & $31(23)$ & \multirow{2}{*}{135} \\
\hline & Married & $104(77)$ & \\
\hline \multirow{3}{*}{ Work shift } & Morning shifts & $7(5.2)$ & \multirow{3}{*}{135} \\
\hline & Two shifts & $123(91.1)$ & \\
\hline & Three shifts & $5(3.7)$ & \\
\hline \multirow{6}{*}{ Hospital ward } & Emergency and burn units & $19(14.1)$ & \multirow{6}{*}{135} \\
\hline & Dialysis unit, CCU, ICU, and NICU & $43(31.9)$ & \\
\hline & Internal unit, neurosurgery department, and infectious diseases unit & $36(26.7)$ & \\
\hline & Surgery and orthopedics section & $20(14.8)$ & \\
\hline & Neonatal ward & $9(6.7)$ & \\
\hline & Coronary care unit & $8(5.9)$ & \\
\hline \multirow{2}{*}{ Settlement status } & Native & $129(95.6)$ & \multirow{2}{*}{135} \\
\hline & Non-native & $6(4.4)$ & \\
\hline
\end{tabular}

intervention showed a significant difference in the intervention group $(P>0.001)$, while there was no significant difference in these variables before and after the intervention in the control group $(P>0.05)$ (Table 3).

The independent samples t-test was used to compare the groups in terms of the study variables before and after the intervention. Before the intervention, major anxiety was reported in the intervention group in comparison with the control group. Also, the analysis showed that the scores of emotional intelligence were significantly different between the groups after training (Table 4).

The Chi-square test results showed the

Table 2. The mean $\pm \mathrm{SD}$ of qualitative variables in the study population

\begin{tabular}{lc}
\hline Variables & Mean \pm SD \\
\hline Age & $32.11 \pm 6.68$ \\
Work experience & $7.24 \pm 5.72$ \\
State anxiety score before the intervention & $42.98 \pm 7.76$ \\
Trait anxiety score before the intervention & $38.00 \pm 8.36$ \\
Emotional intelligence score before the intervention & $327.02 \pm 33.44$ \\
State anxiety score after the intervention & $40.11 \pm 7.70$ \\
Trait anxiety score after the intervention & $36.40 \pm 8.75$ \\
Emotional intelligence score after the intervention & $339.50 \pm 39.11$ \\
\hline
\end{tabular}

homogeneity of the two groups in terms of gender (with a similar distribution). Also, the analysis showed no significant difference in the distribution of the two groups regarding educational level; in fact, the two groups were found to be homogenous. In addition, the non-parametric test (Chi-square) results showed that the two groups were homogeneous in terms of marital status and settlement status. On the other hand, as Chi-square test could not be used for the evaluation of work shifts and units (given the variations), the Monte Carlo open sampling method was applied to determine the exact P-value; these two variables (work shifts and units) were not significant in our research.

State anxiety in the control $(P<0.001 ; \mathrm{r}=-0.476)$ and intervention $(P=0.024 ; \quad \mathrm{r}=-0.320)$ groups significantly decreased as emotional intelligence increased. There was a reverse and significant relationship between the changes in trait anxiety and emotional intelligence in the control $(P=0.142 ; \mathrm{r}=-$ $0.190)$ and intervention $(P=0.006 ; \mathrm{r}=-0.384)$ groups. In addition, there was a reverse and significant association between total anxiety and emotional intelligence in the control $(P=0.023 ; \mathrm{r}=-0.290)$ and intervention $(P=0.005 ; \mathrm{r}=-.394)$ groups.

The covariance of emotional intelligence showed 
Table 3. The comparison of study variables before and after the intervention in the groups using paired t-test

\begin{tabular}{|c|c|c|c|}
\hline & & Mean & P-value \\
\hline \multirow{8}{*}{ Intervention group $(\mathrm{n}=62)$} & Total anxiety score before the intervention & $84.95 \pm 15.76$ & \multirow{2}{*}{$*<0.00$} \\
\hline & Total anxiety score after the intervention & $72.98 \pm 13.55$ & \\
\hline & State anxiety score before the intervention & $45.17 \pm 7.55$ & \multirow{2}{*}{$*<0.001$} \\
\hline & State anxiety score after the intervention & $35.10 \pm 7.32$ & \\
\hline & Trait anxiety score before the intervention & $39.77 \pm 9.07$ & \multirow{2}{*}{$*<0.001$} \\
\hline & Trait anxiety score after the intervention & $34.06 \pm 7.52$ & \\
\hline & Emotional intelligence score before the intervention & $319.6 \pm 34.02$ & \multirow{2}{*}{$*<0.001$} \\
\hline & Emotional intelligence score after the intervention & $350.11 \pm 29.67$ & \\
\hline \multirow{8}{*}{ Control group $(\mathrm{n}=73)$} & Total anxiety score before the intervention & $77.61 \pm 13.77$ & \multirow{2}{*}{0.39} \\
\hline & Total anxiety score before the intervention & $79.52 \pm 15.68$ & \\
\hline & State anxiety score before the intervention & $43.12 \pm 7.92$ & \multirow{2}{*}{0.144} \\
\hline & State anxiety score after the intervention & $43.12 \pm 7.50$ & \\
\hline & Trait anxiety score before the intervention & $36.49 \pm 7.99$ & \multirow{2}{*}{1.0} \\
\hline & State anxiety score after the intervention & $36.39 \pm 9.27$ & \\
\hline & Emotional intelligence score before the intervention & $333.76 \pm 31.62$ & \multirow{2}{*}{0.56} \\
\hline & Emotional intelligence score after the intervention & $330.49 \pm 43.54$ & \\
\hline
\end{tabular}

Table 4. The comparison of study variables between the intervention $(n=62)$ and control $(n=73)$ groups before and after the intervention using independent two-sample t-test

\begin{tabular}{|c|c|c|c|c|c|c|}
\hline \multicolumn{7}{|c|}{ Statistics } \\
\hline & Groups & $\mathbf{N}$ & Mean & SD & Standard error & P-value \\
\hline \multirow{2}{*}{ State anxiety score before the intervention } & Intervention & 62 & 45.1774 & 7.52506 & 0.95911 & \multirow{2}{*}{0.002} \\
\hline & Control & 73 & 41.1233 & 7.50175 & 0.87801 & \\
\hline \multirow{2}{*}{ Trait anxiety score before the intervention } & Intervention & 62 & 39.7742 & 9.07423 & 1.15243 & \multirow{2}{*}{0.027} \\
\hline & Control & 73 & 36.4932 & 7.99674 & 0.93595 & \\
\hline \multirow{2}{*}{ Emotional intelligence before the intervention } & Intervention & 62 & 31.90806 & 34.02280 & 4.32090 & \multirow{2}{*}{0.010} \\
\hline & Control & 73 & 333.7671 & 31.62652 & 3.70160 & \\
\hline \multirow{2}{*}{ Trait anxiety before the intervention } & Intervention & 62 & 38.9194 & 7.32008 & 0.92965 & \multirow{2}{*}{0.098} \\
\hline & Control & 73 & 41.1233 & 7.92490 & 0.92754 & \\
\hline \multirow{2}{*}{ Trait anxiety after the intervention } & Intervention & 62 & 34.0645 & 7.52454 & 0.95562 & \multirow{2}{*}{0.003} \\
\hline & Control & 73 & 38.3973 & 9.27473 & 1.08552 & \\
\hline \multirow{2}{*}{ Emotional intelligence after the intervention } & Intervention & 62 & 350.1129 & 29.67535 & 3.76877 & \multirow{2}{*}{0.003} \\
\hline & Control & 73 & 330.4932 & 43.84636 & 5.13183 & \\
\hline \multirow{2}{*}{ Total anxiety after the intervention } & Control & 73 & 79.5205 & 15.68271 & 1.83552 & \multirow{2}{*}{0.01} \\
\hline & Intervention & 62 & 72.9839 & 13.55921 & 1.72202 & \\
\hline \multirow{2}{*}{ Total anxiety before the intervention } & Control & 73 & 77.6164 & 13.77864 & 1.61267 & \multirow{2}{*}{0.005} \\
\hline & Intervention & 62 & 84.9516 & 15.76095 & 2.00164 & \\
\hline
\end{tabular}

that stress significantly decreased by improving emotional intelligence through training. In our analysis, the effects of emotional intelligence on total, state, and trait anxiety were evaluated under the condition that anxiety scores were normalized with respect to their baseline values in the pretest.

\section{Discussion}

The results of the present study showed that nurses employed in Sabzevar hospitals are young. A significant decline was observed in anxiety among nurses after evaluating the components of emotional intelligence. 
This indicates that nurses experience more stress in the first years of employment, although this anxiety can be mediated by education and training.

In the present study, the mean age of the participations was 32.11 years, which seems to be lower in comparison with similar research (19). The results of this study showed that the variances were similar in the two groups. In other words, the groups were homogeneous, and we could neglect the probable role of these factors in anxiety; therefore, the educational effects of emotional intelligence on anxiety could be directly measured. Also, the results showed that demographic characteristics had no effects on anxiety, as confirmed in recent studies (24).

In addition, comparison of emotional intelligence scores in the groups showed a significant increase following training, i.e., proper emotional intelligence training could improve emotional intelligence, as similarly reported in previous research (19). The present results were in consistence with similar previous studies, which showed that emotional intelligence training could decrease anxiety $(19,25-27)$.

According to the results of the current research, the reactive effects of emotional intelligence training on changes in anxiety scores were significant; also, the actual effects were found to be major. In fact, by controlling the effects of other variables, a one-unit increase in emotional intelligence could lead to a 0.221 unit decline in total anxiety in the control group and a 7.186-unit decline in the intervention group.

Trait anxiety was found to be similar to total anxiety. In fact, a one-unit increase in emotional intelligence could decrease descriptive anxiety by 4.134 units in the intervention group. Also, in the intervention group, a one-unit increase in emotional intelligence could decrease trait anxiety by 0.141 units. State anxiety was similar to total and trait anxiety. A one-unit increase in emotional intelligence could decrease state anxiety in the intervention group by 2.532 units. Also, in the intervention group, as emotional intelligence increased by one unit, the average trait anxiety decreased by 0.18 units; this finding was in consistence with similar research (25-27).

\section{Limitations}

The present study had several limitations. First, it was not possible to assess, measure, or control the educational content of the sessions in the intervention group. Second, the subjects' self-report on anxiety might have affected the results. Third, the high number of items in the questionnaires was a common problem in the process of questionnaire adaptation.

\section{Conclusion}

According to the present findings, anxiety in nurses can result in a qualitative and quantitative decline in the provision of healthcare services; in fact, anxiety can lead to unfavorable effects on nurses' social health. Considering the involvement of nurses in healthcare teams, their continuous interaction with the sick, their sensitive caring role, and the importance of stress control in improving the quality of care, emotional intelligence training at the beginning or during nurses' employment could be effective in reducing anxiety and improving the quality of nursing services.

\section{Suggestions}

Considering the effect of emotional intelligence training on reducing nurses' anxiety, this type of training at the beginning of employment or during nursing services is highly recommended.

\section{Conflicts of interest}

The authors declare no conflicts of interest.

\section{Author's contributions}

The authors all made equal contributions to this paper.

\section{Acknowledgements}

This research was derived from a M.Sc. thesis in nursing and midwifery, submitted to Sabzevar University of Medical Sciences. We would like to thank the educational members of the school, the deputy of health, and the head of Vasee Hospital, affiliated to Sabzevar University of Medical Sciences for their contribution to the development 
of the emotional intelligence training workshop. We also extend our gratitude to the participants for their sincere cooperation.

\section{References}

1. Tomar R. A study of emotional intelligence among nurses. Int J Sci Res Edu 2016; 4(4):5204-11.

2. Uddin MT, Islam MT, Ullah MO. A study on the quality of nurses of nurses of government hospitals in Bangladesh. Proc Pakistan Acad Sci 2006; 43(2):121-9.

3. Khaghanizadeh M, Salemi SH, Rahmani R. Effective factors on Job burnout in military nurses. Military Medicine Congress, Tehran, Iran; 2003. P. 290.

4. Krueger P, Brazil K, Lohfeld L, Edward HG, Lewis D, Tjam E. Organization specific predictors of job satisfaction: findings from a Canadian multi-site quality of work life cross-sectional survey. BMC Health Serv Res 2002; 2(1):6.

5. Pflanz S, Sonnek S. Work stress in the military: prevalence, causes, and relationship to emotional health. Mil Med 2002; 167(11):877-82.

6. Sardar MA. Effect of eight weeks of aerobic training on mental health, depression, anxiety, somatic complaints, social dysfunction in male students. [Phd Dissertation]. Ahvaz:Joundi Shapour University; 2008 (Persian).

7. Garcia-Sancho E, Salguero JM, Fernandez-Berrocal P. Relationship between emotional intelligence and aggression: a systematic review. Aggress Violent Behav 2014; 19(5):584-91.

8. Bar-On RE, Parker JD. The handbook of emotional intelligence: Theory, development, assessment and application at home, school and in the workplace. New York: Jossey-Bass; 2000.

9. Summerfeldt LJ, Kloosterman PH, Antony MM, Parker JD. Social anxiety, emotional intelligence, and interpersonal adjustment. J Psychopathol Behav Assess 2006; 28(1):57-68.

10. Mennin DS, Heimberg RG, Turk CL, and Fresco DM. Applying an emotion regulation framework to integrative approaches to generalized anxiety disorder. Clin Psychol 2002; 9(1):85-90.

11. Mennin DS, Turk CL, Heimberg RG, Carmin CN. Focusing on the regulation of emotion: a new direction for conceptualizing and treating generalized anxiety disorder. In: Reinecke MA, Clark DA, editors. Cognitive therapy over the lifespan: theory, research and practice. New York: Wiley; 2004.

12. Sunil K, Rooprai KY. Role of emotional intelligence in managing stress and anxiety at workplace. Proc ASBBS 2009; 16(1):163-72.

13. Siu AF. Trait emotional intelligence and its relationships with problem behavior in Hong Kong adolescents. Personal Individ Dif 2009; 47(6):553-7.

14. Boussiakou LG, Boussiakou IZ, Kalkani EC. Student development using emotional intelligence. Word Trans Engin Technol Edu 2008; 5(1):53.

15. Ciarochi J, Deane FP, Anderson S. Emotional intelligence moderates the relationship between stress and mental health. Personal Individ Dif 2002; 32(2):197-209.

16. Salovey P, Grewal D. The science of emotional intelligence. Curr Direct Psychol Sci 2005; 14(6):281-5.

17. Nooryan K, Gasparyan K, Sharif F, Zoladl M, Moghimi M, Hosseini NA. The effects of emotional intelligence (EI) items education on job related stress in physicians and nurses who work in intensive care unit. Armaghane Danesh 2011; 16(5):472-9.

18. Yousefi R, Namdari Y, Adhamiyan A. A comparison of depression and occupational stress in psychiatrics and non psychiatric unit nurses and official employees. J Urmia Nurs Midwifery Facul 2006; 4(2):80-90 (Persian).

19. Delavar A. statistical method in psychology and Education. Tehran: Payam Nour Publication; 2007.

20. Dehshiri Q. Norms of Bar-on emotional intelligence test on undergraduate students in Tehran University. [Doctoral Dissertation]. Tehran, Iran: Tabatabai University; 2003.

21. Montes-Berges B, Augusto-Landa JM. Emotional intelligence and affective intensity as life satisfaction and psychological well-being predictors on nursing professionals. J Prof Nurs 2014; 30(1):80-8.

22. Hemati Mastak Pak M. Assessment of anxiety level of employed formal nurses in hospitals of Urmia University of Medical Sciences. J Urmia Midwifery Facul 2005; 3(2):110 (Persian).

23. Femandez-Berrocal P, Alcaide R, Extremera N, Pizarro D. The role of emotional intelligence in anxiety and depression among adolescents. Individ Dif Res 2006; 4(1):16-27.

24. Downey LA, Johnston PJ, Hansen K, Schembro R, Stough $\mathrm{C}$, Tuckwell V, et al. The relationship between emotional intelligence and depression in a clinical sample. Eur $\mathrm{J}$ Psychiat 2008; 22(2):93-8.

25. Fernandez-Berrocal P, Salovey P, Vera A, Extremera N, Ramos N. Cultural influences on the relation between perceived emotional intelligence and depression. Int Rev Soci Psychol 2005; 18(1):91-107.

26. Elliott T, Shewchuk RM, Richards JS. Family caregiver problem solving abilities and adjustment during the initial year of the care giving role. J Counsel Psychol 2001; 48(2):223.

27. Shewchuk R, Richards JS, Elliott TR. Dynamic processes in health outcomes among caregivers of patients with spinal cord injuries. Health Psychol 1998; 17(2):125-9.

28. Spielberger CD. State-trait anxiety inventory. $2^{\text {nd }}$ ed. New York: John Wiley \& Sons, Inc; 2010

29. Spielberger CD, Gorsuch RL, Lushene R, Vagg PR, Jacobs GA. Manual for the state-trait anxiety inventory. Palo Alto, CA: Consulting Psychologists Press; 1983.

30. Coleman AM. A dictionary of psychology. $3^{\text {nd }}$ ed. Oxford, United Kingdom: Oxford University Press; 2008. 